\title{
Revisión de la jurisprudencia administrativa de la Superintendencia de Valores y Seguros en relación a la oferta pública y privada de valores en Chile
}

\author{
Review of the Administrative Jurisprudence of the Superintendency of Securities \\ and Insurance in relation to the Public and Private Offering of Securities in Chile
}

\author{
Mario Valderrama Venegas \\ Ferrada Nehme, Chile
}

\begin{abstract}
RESUMEN Este artículo presenta un panorama de la interpretación administrativa de la Superintendencia de Valores y Seguros en relación al concepto de oferta pública/privada de valores, conforme a la Ley 18.045 de Mercado de Valores. Además, realiza algunos breves comentarios al régimen de excepción de inscripción en el registro de valores. La presente contribución tiene por objeto dar mayor claridad respecto a conceptos básicos para la aplicación de la Ley 18.045 en Chile.
\end{abstract}

PALABRAS CLAVE Oferta pública de valores, oferta privada de valores, valores.

\begin{abstract}
This article presents an overview of administrative interpretations of the Chilean Superintendence of Securities and Insurance related to the meaning of «public/ private offering» of securities, according to Law No. 18,045, Securities Market Law. In addition, it makes some brief comments to the safe harbor regime in Chile. The purpose of this paper is to clarify some basic concepts for the application and interpretation of the Chilean Securities Market Law.
\end{abstract}

KEYWORDS Public offering, private offering, securities.

\section{Antecedentes}

La Ley 18.045 de Mercado de Valores (LMV) establece como núcleo central de la reglamentación de ese mercado el concepto de oferta pública de valores (Trucco, 1982: 112), por lo que resulta trascendental comprenderlo detalladamente, ya que de eso depende: i) la propia aplicación de la Ley y su regulación; y ii) la supervisión de la Superintendencia de Valores y Seguros (SVS). 
Lo anterior es claro ya a partir del artículo 1 de la Ley 18.045, que establece:

A las disposiciones de la presente ley queda sometida la oferta pública de valores y sus respectivos mercados e intermediarios [...] aplicándose este cuerpo legal a todas aquellas transacciones de valores que tengan su origen en ofertas públicas de los mismos o que se efectúen con intermediación por parte de corredores o agentes de valores.

Las transacciones de valores que no sean de aquellas a que se refiere el inciso primero del presente artículo, tendrán el carácter de privadas y quedarán excluidas de las disposiciones de esta ley, excepto en los casos en que ésta se remita expresamente a ellas.

A su turno, el artículo segundo de la misma Ley establece:

Corresponderá a la Superintendencia de Valores y Seguros, en adelante la Superintendencia, vigilar el cumplimiento de las disposiciones de la presente ley, de acuerdo con las facultades que se le confieren en su ley orgánica y en el presente cuerpo legal.

Como complemento de lo anterior, el artículo 6 de la Ley 18.045 establece:

Sólo podrá hacerse oferta pública de valores cuando éstos y su emisor hayan sido inscritos en el Registro de Valores.

La inscripción de las acciones a que se refiere la letra c) del artículo 5, deberá efectuarse dentro de los sesenta días siguientes a la fecha en que se haya cumplido alguno de los requisitos allí mencionados.

Finalmente, la oferta pública de valores no inscritos, suspendidos o cancelados del señalado Registro se encuentra sujeta a sanción penal, conforme a la letra a) del artículo 60 de la LMV:

Sufrirán las penas de presidio menor en cualquiera de sus grados: a) Los que hicieren oferta pública de valores sin cumplir con los requisitos de inscripción en el Registro de Valores que exige esta ley o lo hicieren respecto de valores cuya inscripción hubiere sido suspendida o cancelada.

Finalmente, este trabajo presentará el desarrollo legislativo, regulatorio y de interpretación administrativa del concepto de valor y de oferta pública, dedicando secciones separadas para cada uno, para luego hacer un repaso del régimen de excepción establecido por la SVS en virtud de sus facultades normativas establecidas en el inciso segundo del artículo 4 de la LMV.

\section{Concepto de valor}

Nuestra LMV define valor en su artículo 3 como:

cualesquiera títulos transferibles incluyendo acciones, opciones a la compra y venta de acciones, bonos, debentures, cuotas de fondo mutuos, planes de ahorro, efectos de comercio y, en general, todo título de crédito o inversión. 
De esta manera, la LMV establece un concepto general de valor y señala como ejemplos cierto tipo de éstos, no existiendo un catálogo cerrado de valores, sino que cualquier instrumento financiero que cumpla con los elementos de la definición general establecida en el artículo 3, ya citado, es considerado valor.

En el mismo sentido, de acuerdo a la Historia Fidedigna del establecimiento de la LMV, en relación al término valores se indicó:

También se define el término «valores» en una forma amplia de modo que los nuevos títulos transferibles de crédito a propiedad que se puedan crear en el futuro mediante técnicas financieras más desarrolladas también caigan bajo la esfera de la ley (Biblioteca del Congreso Nacional, 2013: 72).

De esta forma, para la LMV lo relevante es el acto de la oferta, y que ésta tenga como veremos más adelante- el carácter de pública y no el establecer un catálogo de «valores de oferta pública». En este punto, cabe destacar que ha existido jurisprudencia de la SVS en sentido contrario. En el Oficio Ordinario 6.306 de 1999, se señaló, en resumen, que un bono sólo puede emitirse y colocarse conforme a las reglas de la LMV, es decir, sólo puede ofrecerse previo a su inscripción en el Registro de Valores y, en consecuencia, no es posible, bajo nuestra legislación, la oferta privada de bonos.

En relación a esto, en mi opinión, incluso previo a la dictación de la Norma de Carácter General núm. 336 de 2012 (NCG 336), ha sido posible la colocación privada de bonos, y no son aplicables en ese caso las obligaciones que establece la LMV. El mencionado Oficio Ordinario simplemente no da cuenta del tenor literal del artículo 103 de la LMV que establece que «La oferta pública de valores representativos de deuda cuyo plazo sea superior a un año, sólo podrá efectuarse mediante bonos y con sujeción a las disposiciones generales establecidas en la presente ley y a las especiales que se consignan en los artículos siguientes»; ni tampoco del artículo 131 de la LMV que establece:

Sin perjuicio de lo establecido por el artículo 103 de esta ley, la oferta pública de valores representativos de deuda cuyo plazo no sea superior a 36 meses, también podrá efectuarse mediante la emisión de pagarés u otros títulos de crédito, con sujeción a las disposiciones de esta ley y a los requisitos que establezca la Superintendencia mediante la dictación de instrucciones de carácter general.

Incluso nuestra LMV permite un «bono corto», es decir, con vencimiento menor a un año, en el inciso final del artículo 131: «Sin perjuicio de lo establecido en el inciso primero, la emisión de títulos de deuda regulados por este artículo podrá también efectuarse bajo la forma y disposiciones del Título XVI de esta ley». Es por ello que un instrumento financiero de deuda denominado «bonos» no reviste ninguna característica particular bajo la LMV, a menos que se haga oferta pública de éste, por lo que se puede ofrecer privadamente (Guerrero, 2002).

Por otra parte, la jurisprudencia administrativa de la SVS, emitida conforme a la potestad establecida en la letra a) del artículo 4 del Decreto Ley 3.538 de 1980, ha iden- 
tificado los elementos que requiere los instrumentos financieros para ser considerados «valor» (Oficio Ordinario, Superintendencia de Valores y Seguros 9.100 de 2015 y 3.502 de 2017), haciendo un breve análisis del artículo 3 de la LMV, indicando que conforme dicha definición legal tiene los siguientes elementos, a saber:

i) Se debe tratar de un título.

ii) representativo de crédito o inversión, es decir, de sus características intrínsecas se deriva una relación jurídica de acreedor/deudor o una relación jurídica inversionista/derechos sobre rendimientos de capital, derechos sobre flujos que se establecen de manera anticipada en el primer caso y, en el segundo caso, eventuales, en la medida que existan rendimientos positivos sobre la inversión. Dichos derechos sobre flujos son independientes de alguna acción por parte de su titular, sea la aplicación de trabajo y tiempo de su parte.

iii) De libre circulación, es decir, por regla general su transferencia no requiere del consentimiento de terceros, ni de otras formalidades.

iv) De carácter autónomo, es decir, el primer titular del documento lo adquiere en forma originaria por lo que su derecho no deriva del emisor del documento, sino que se origina nuevamente en el adquirente. El primer titular no es sucesor del sujeto que emitió el título. En consecuencia, el portador ejerce un derecho propio distinto e independiente de las relaciones jurídicas del emisor del título.

iv) Literalidad, es decir, que, por regla general, su contenido, extensión y modalidades dependen exclusivamente del tenor del título, cualquier modificación disminución o mutación debe resultar de los términos textuales del título.

Como puede apreciarse, el concepto de valor es estricto, y no comprende la totalidad de los instrumentos financieros o de inversión, lo cual excluye del concepto de valor a las monedas físicas (Oficio Ordinario SVS 21.775 de 2010; Trucco, 1982: 115) o virtuales copias cedibles de facturas, ciertos contratos financieros, como contratos de derivados (salvo las opciones de compra y venta de acciones de las sociedades anónimas, por expreso mandato legal), entre los que se encuentran opciones y futuros (Oficios Ordinarios de SVS 19.407 de 2014 y 2.950 de 1995), forwards, swaps, contratos por diferencia (Oficios Ordinarios de SVS 14.891 de 2012; 21.775 de 2010 y 2.053 de 2008), y cualquier otro contrato o instrumento financiero (o no financiero).

Tampoco son considerados valores por la SVS las cuentas de ahorro, ya que ellas no cumplen con el requisito de libre circulación o cesibilidad y no son títulos de crédito; los aportes previsionales voluntarios, APVC y depósitos convenidos (todos ellos con fines previsionales), ya que no son transferibles; depósitos a plazo, ya que no cumplen con el requisito de libre circulación o cesibilidad, ya que por su naturaleza, sólo pueden transferirse conforme a las formalidades de la cesión de créditos; libretas de ahorro, ya que no cumplen con el requisito de libre circulación o cesibilidad, y no son títulos de crédito; saldos de cuenta corriente, desde el momento que no son títulos de crédito ni de inversión, y no cumplen con el requisito de libre circulación o cesibilidad; seguro de vida con ahorro y seguros en general, ya que son contratos y no constituyen títulos de crédito ni de inversión; los cheques no son considerados valor ya que no es un título de 
inversión. En definitiva, no es valor cualquier instrumento o cosa que no cumpla con alguna de las características señaladas arriba.

Lo anterior, según hemos dicho, tiene una gran importancia, ya que, de carecer un instrumento financiero de alguna de dichas características, se encuentran excluidos de la aplicación de la LMV y la supervigilancia de la SVS (Pavez, 2016).

\section{Concepto de oferta pública y régimen de excepción de registro}

Una vez revisado el concepto de valor, resulta necesario repasar el concepto de oferta pública, establecido en el artículo 4 de la Ley de Mercado de Valores, que establece: «Se entiende por oferta pública de valores la dirigida al público en general o a ciertos sectores o a grupos específicos de éste».

En este punto resulta necesario entender el concepto de oferta. La LMV no define oferta de manera específica. Sin embargo, la doctrina del derecho civil la define como acto jurídico unilateral por el cual una persona propone otra celebrar una determinada convención (Vial, 2009: 63) o como una manifestación de voluntad de carácter unilateral, recepticia, seria, precisa, completa y dirigida a una persona determinada (Segura, 2006). La oferta, para que forme el consentimiento, debe ser completa, lo que significa que se realice de forma que sea suficiente la simple aceptación de la persona a la que es dirigida para que se perfeccione la convención. Si se trata de contratos reglamentados por el legislador, la oferta debe contener los elementos esenciales de éste. En el caso de no ser completa, se trataría de una tratativa preliminar (Vial, 2009: 64).

Sin embargo, de los requisitos antes señalados se desprende que dicho concepto tradicional tiene tres limitaciones a objeto de interpretar la LMV, que son las siguientes. Primero, la interpretación que realiza la SVS a la LMV es una interpretación de carácter administrativo, lo que significa que es en base a sus fines específicos. De esta forma, la administración tiene un mandato de optimización en relación a los fines específicos de las normas que por ley está obligada a interpretar, y, en el caso de la LMV, uno de sus pilares centrales es proteger al público inversionista de la distribución de riesgos de mercado, para efectos de poder generar confianza en éste. Segundo, para la doctrina del derecho civil una oferta es obligatoria cuando tiene un destinatario específico, lo cual precisamente de desmarca del fenómeno de la oferta de valores de carácter indiscriminado, con destinatario indeterminado y de carácter masivo. Tercero, dicho concepto tradicional de oferta y sus requisitos no se ajustan a la jurisprudencia de la SVS en la materia. En este punto, cabe resaltar que la SVS ha entendido el concepto de oferta de valores de manera más amplia, como toda invitación genérica a invertir en valores. Esto se puede observar en la resoluciones exentas 468 y 469 de 2009, en las que se sancionó con censura al Club Deportivo Municipal Iquique SADP y con una multa a beneficio fiscal de UF 1.000 a Euroamérica Corredores de Bolsa SA, respectivamente, por infracción, entre otras disposiciones, del artículo 4 de la LMV, al invitar al público general, a través de diversos medios de comunicación, incluyendo avisos en el sitio web del mencionado club e insertos en diarios de amplia circulación, a acercarse a las 
oficinas del intermediario para aportar capital para la constitución de una futura sociedad concesionaria, a través de amplia publicidad de diversas frases de propaganda, tales como «Ésta es la nuestra [...] Para llevar a Municipal Iquique a las grandes ligas... únete a nosotros [...] Juntos vamos a participar en la Oferta Privada de Acciones [...] Para mayor información, acércate a las oficinas de Euroamérica Corredora de Bolsa».

En otro orden de ideas, dado el tenor del artículo 4 de la LMV, se trata de una oferta de enajenación de valores. En relación a la materia, la SVS ha interpretado que la oferta no es exclusivamente de venta, sino que también de otro tipo de operaciones donde no existe intercambio de flujo de dinero; por ejemplo, la oferta de canje de acciones emitidas por una sociedad anónima absorbente respecto de los accionistas de una sociedad anónima abierta, en virtud de un proceso de fusión por absorción, es una oferta pública de valores, en consideración a que la oferta de canje, dirigida a un número relevante de accionistas, se enmarca en la definición del artículo 4 de la LMV (ver Oficios Ordinarios SVS 5.738 de 2011 y 3.283 de 2015). El mismo criterio es aplicable a la distribución de acciones producto de la división de una sociedad anónima abierta. También es una oferta pública de valores aquella oferta gratuita de éstos.

En consideración al texto del artículo 4, ya señalado, y previo a la dictación de la Norma de Carácter General 336 de 2012, no resultaba sencillo determinar si en un caso específico nos encontramos frente a una oferta pública de valores, especialmente cuando una oferta de valores se entendía dirigida a ciertos sectores o grupos específicos del público general (Trucco, 1983: 80; Jara, 2001: 105; Ried, 2006). Para la SVS, un caso claro de oferta pública de valores era aquella realizada mediante su difusión a través de medios de comunicación masivos o en las que participaran en los esfuerzos de colocación de algún intermediario de valores, conforme al artículo 1 de la LMV. Sin embargo, existían casos mucho más difíciles de resolver. Por ejemplo, una sociedad anónima cerrada emite cien pagarés al portador y se los ofrece directamente en reuniones uno a uno a diez inversionistas no calificados: pareciera ser que, dado el esfuerzo de comercialización individual y el número muy reducido de inversionistas, estaríamos frente a una oferta privada de valores. ¿Cambia en algo la conclusión si sostiene reuniones uno a uno con cien inversionistas no calificados? Pareciera que existe la posibilidad de considerarla pública, debido al mayor número de inversionistas. ¿Cambia en algo la conclusión si esas diez personas son gerentes de inversión de inversionistas institucionales? Pareciera ser que, dada las características de los destinatarios de la oferta, se trata de una dirigida a un grupo específico del público general, por lo que pudiera entenderse como pública. En definitiva, a falta - en esa época- de criterios regulatorios, cualquier respuesta a estas preguntas no correspondía a la aplicación de una regla clara, sino que correspondía al criterio aplicable caso a caso por la SVS.

Es por ello que la NCG 336, dictada en virtud del inciso segundo del artículo 4 de la LMV, es un avance importante en esta materia, ya que permite tener lo que se conoce en otros ordenamientos jurídicos como safe harbor, estableciendo claridad de lo que la SVS entiende como oferta privada de valores, o, dicho de otro modo, establecer aquellas ofertas de valores que no constituyen ofertas públicas. 
Así, conforme a la NCG 336, las ofertas de valores que no constituirán ofertas públicas de valores son aquellas ofertas sobre un mismo valor, que copulativamente: i) cumplan con las obligaciones de información y resguardo establecidas en las secciones III y IV de la NCG 336; ii) no se realicen mediante medios masivos de difusión; y iii) sean dirigida a destinatarios elegibles. Existe un tipo de oferta que no requiere este último requisito: aquellas ofertas de valores que traten sobre instrumentos cuyo valor unitario ascienda al menos al equivalente a 5.00o Unidades de Fomento, ya que el adquirente de un instrumento con esas características se entiende como inversionista calificado.

\section{Conclusiones}

En relación a la NCG 336 y el régimen de oferta privada de valores, es posible concluir:

1) Regula de manera más estricta el resale o reventas, en aquella oferta de valores dirigidos a no más de 250 inversionistas calificados de aquellos señalados en los números 7 y 8 de la Sección II de la Norma de Carácter General 216 de 2008 (pudiendo incluirse dentro de los 250 destinatarios, un máximo de 50 inversionistas que no tengan la calidad de inversionista calificado), mediante una o varias ofertas sucesivas, en el plazo de 12 meses contados desde la primera de las ofertas. Por ello, dentro de esos 12 meses contados desde la primera oferta, todas las ofertas de venta y de reventa se consideran para los efectos de calcular el máximo de destinatarios de la oferta, por lo que posteriores ofertas o reventa pueden, eventualmente, en total, exceder el número máximo de destinatarios y, de esa forma, convertirse en oferta pública de valores. En los demás casos, cada oferta de venta o reventa deberá cumplir individualmente la NCG 336.

2) No es per se oferta pública de valores toda oferta de valores que no cumpla alguno de los requisitos de la NCG 336; por ejemplo, una oferta de valores dirigida únicamente a inversionistas no calificados será o no privada dependiendo de la concurrencia de alguno de los criterios expuestos por la jurisprudencia de la SVS, especialmente el criterio de difusión masiva. Sin perjuicio de lo anterior, en consideración al criterio cuantitativo establecido en la misma NCG 336, resulta relativamente pacífico concluir que una oferta de valores dirigida a 50 o menos destinatarios no calificados mediante cartas, correos electrónicos y otras comunicaciones, físicas o electrónicas, que estén dirigidas exclusivamente a una persona determinada y debidamente individualizada en la misma comunicación, llamados telefónicos, reuniones, entrevistas personales y sistemas electrónicos de acceso restringido no correspondería a una oferta pública de valores.

3) El eje de esta NCG 336 es, en consistencia con nuestro marco legislativo, la oferta pública de valores. Lo anterior, hace irrelevante para un oferente la cantidad de valores colocados efectivamente. De esta forma, imaginemos que se emiten mil pagarés por parte de una persona jurídica, se la ofrece a 500 personas, de las cuales sólo 30 los aceptan. Para los efectos de la NCG 336 y la LMV, nos encontramos en presencia de una oferta pública de valores.

4) Para ser considerado como medio masivo de difusión (entre otros — según 
establece la NCG 336- de similar naturaleza o alcance, la prensa, radio, televisión e internet), éstos deben ser de acceso público en o desde Chile, sin importar el lugar donde sean producidos o desde donde sean emitidos. En este punto resulta válido aclarar que dicha definición se encuentra limitada por el principio de territorialidad de la ley chilena, por lo que para ser considerado como una oferta pública de valores bajo la LMV deberá existir una conducta desplegada que tenga influencia directa en el mercado de valores chileno, teniendo dicha oferta su origen en Chile o sea dirigida a residentes en Chile.

5) Dado lo señalado anteriormente, y la obligación de acreditar el cumplimiento de las obligaciones establecidas en la letra c) de la Sección IV de la NCG 336, ante cualquier requerimiento de la SVS, es de cargo del oferente de valores mantener los controles adecuados para poder verificar que efectivamente se cumpla dicha norma. Lo anterior implica, en términos prácticos, llevar registros detallados de todo esfuerzo de comercialización de valores al amparo de dicha norma, incluyendo el contenido de estas comunicaciones, sea que dicho esfuerzo sea realizado telefónicamente, a través de correos electrónicos, entre otros, y además la acreditación de los destinatarios de la(s) oferta(s) como inversionistas calificados.

6) Conforme al artículo 1 de la LMV, que establece que se aplica dicho cuerpo legal a «todas aquellas transacciones de valores [...] que se efectúen con intermediación por parte de corredores de bolsa o agente de valores» $y$ el inciso segundo agrega que las «transacciones de valores que no sean de aquellas a que se refiere el inciso primero del artículo 1 ya citado, se entienden privadas»; por lo que la NCG 336 no resulta aplicable a dichos intermediarios, dado que la LMV las califica de públicas per se todas las transacciones de valores que ellos intermedien.

\section{Referencias}

Biblioteca del Congreso Nacional (2013). Historia de la Ley 18.045, de Mercado de Valores. Disponible en http://bit.ly/2vkR6AK.

Guerrero, Roberto (2002). «La oferta privada de bonos: Una errada interpretación administrativa». Revista Chilena de Derecho, 29 (3): 583-591. Disponible en http:// bit.ly/2vlbBNE.

Jara Amigo, Rony (2001). «Oferta pública y privada de valores en el mercado norteamericano: Una visión comparada». Revista Chilena de Derecho, 28 (1): 87-111. Disponible en http://bit.ly/2vUwpiG.

Pavez, Carlos (2016). Presentación para la Comisión especial Investigadora de los organismos públicos competentes en relación al eventual fraude de empresas de inversiones. Disponible en http://bit.ly/2vU3xnQ.

Ried, José Miguel (2006). «El caso Almacenes París. Algunas consideraciones en torno al concepto de oferta pública de valores. Comentario a oficio de Superintendencia de Valores y Seguros, 3 de marzo de 2005». Revista Chilena de Derecho, 33 (1): 149158. Disponible en http://bit.ly/2vlba6a. 
Segura, Francisco (2016). Algunas cuestiones actuales en torno a la oferta y la aceptación. Disponible en http://bit.ly/2g1FyRo.

Trucco, Eduardo (1982). «Aplicación de la Ley 18.045 sobre Mercado de Valores». Revista de Derecho Económico, No 56-57:111-128. DOI: 10.5354/0719-7462.1982.42030.

-. (1983). «Delitos económicos especíicos». Revista de Derecho Económico, 60-61: 7784. DOI: 10.5354/0719-7462.1983.42016.

VIAL, Víctor (2009). Teoría del acto jurídico. 5. edición. Santiago: Editorial Jurídica.

\section{Sobre el autor}

Mario Valderrama Venegas es abogado. Magíster en Derecho de los Negocios por la Universidad Adolfo Ibáñez. Actualmente dirige el Área Corporativa del Estudio Ferrada Nehme. Se desempeñó como Jefe de Gabinete y Abogado Jefe de la Fiscalía de Valores, Superintendencia de Valores y Seguros. Su correo electrónico es mario. valderrama.v@gmail.com. 
